\title{
A novel 8.7-kb mitochondrial genome deletion accurately detects endometriosis in the plasma of symptomatic women
}

\author{
Andrew Harbottle ${ }^{1}$, Andrea Maggrah ${ }^{1}$, Robert Usher ${ }^{1}$, Elise Desa ${ }^{1}$ \& Jennifer M Creed*,1 \\ ${ }^{1}$ MDNA Life Sciences Inc., 2054 Vista Parkway, Ste 400, West Palm Beach, FL 33411, USA \\ *Author for correspondence: j.creed@mdnalifesciences.com
}

\begin{abstract}
Aim: To evaluate an 8.7-kb mitochondrial DNA (mtDNA) deletion as a potential biomarker of endometriosis. Materials \& methods: We tested the diagnostic accuracy of the $8.7-\mathrm{kb}$ deletion real-time PCR assay using 182 prospectively collected blood samples from females presenting with symptoms of endometriosis in a case-control format. Results: The assay differentiated between endometriosis and controls (area under curve: $0.74-0.89)$ with a statistically significant difference $(p<0.05)$ in 8.7-kb deletion levels measured for all disease subtypes and stages. No correlation was seen between $8.7-\mathrm{kb}$ deletion levels and participant or specimen age, hormone status or menstrual phase. Conclusion: The diagnostic accuracy of the 8.7-kb deletion for endometriosis suggests potential utility in the clinic to improve patient management.
\end{abstract}

First draft submitted: 11 October 2019; Accepted for publication: 7 November 2019; Published online: 29 November 2019

Keywords: biomarker $\bullet$ diagnostic accuracy $\bullet$ DNA deletion $\bullet$ endometriosis $\bullet$ mitochondrial DNA $\bullet$ molecular diagnostic $\bullet$ mtDNA $\bullet$ real-time PCR

Endometriosis occurs when endometrial tissue grows outside the uterus and affects approximately $5-10 \%$ of women of reproductive age, with infertility, a symptom of this condition, reported in $30-50 \%$ of patients [1-8]. Symptoms vary in severity, and include pelvic pain, painful menstrual cramps, discomfort during intercourse and chronic tiredness [9]. This painful condition can significantly impact a patient's quality of life, resulting in days off work and loss of productivity [10]. Endometriosis is associated with substantial costs and has a comparable economic burden to that of other chronic diseases such as diabetes, Crohn's disease and rheumatoid arthritis [11].

Laparoscopic surgery followed by histopathological confirmation is needed to make a definitive diagnosis [5,12], but this is invasive and patients are, understandably, reluctant to go through with the procedure causing further delays in diagnosis and treatment [13]. There is, however, an increasingly important role for a clinical or presumptive diagnosis of disease, which can support the initiation of treatment and management strategies to control symptoms [14]. Ballard et al. [15] have reported the value of a diagnosis of endometriosis to the patient beyond the availability of treatment options including legitimizing access to social support, excusing absences from work and social events due to symptoms and providing a language with which to communicate about their disease. These can be considered a benefit of both presumptive and surgical diagnoses.

Increasingly, molecular biomarkers are being used in many areas of medicine to detect and manage diseases [16-22]. However, to date, an endometriosis-specific biomarker has not been found that can be used successfully in clinical practice [23-25]. Nonstandard procedures for sample collection and data analysis have hampered researchers' ability to find such a biomarker. However, recent efforts to harmonize sample collection and storage, analysis methods and the reporting of data, encouraged by the publication of the World Endometriosis Research Foundation EPHect Protocols [26], have contributed toward the development of disease-specific assays.

In this paper, we describe the investigation of an $8.7-\mathrm{kb}$ mitochondrial DNA (mtDNA) deletion as a potential biomarker for diagnosing endometriosis, including an initial assessment of diagnostic accuracy followed by an evaluation of disease specificity by comparing the biomarker's frequency in plasma from women with: endometriosis and symptomatic controls, and endometrial cancer, ovarian cancer and breast cancer. 


\section{Methods}

\section{Diagnostic accuracy: participants \& sample collection}

This was a case-control study in which residual plasma samples prospectively collected from women aged 18 years and over (until menopause) who were not pregnant and were scheduled to undergo laparoscopy for suspected endometriosis because of pelvic pain (symptomatic controls and endometriosis cases) or tubal ligation (asymptomatic controls) were used. This study was conducted as part of the EndOx study at Oxford Endometriosis CaRe Centre, John Radcliffe Hospital, University of Oxford, UK.

The collection, anonymization and processing of samples and data were as previously reported [26-31]. Study conduct, relevant authority approvals (Oxfordshire REC A, 09/H0604/58) and consent procedures were also as previously reported [26-31].

\section{Diagnostic accuracy: participant populations/cohorts}

Collected samples were classified as either control or case samples. The control group comprised: asymptomatic controls, which were specimens collected from participants who underwent scheduled tubal ligation without clinical suspicion of endometriosis, and who had surgically confirmed absence of endometriosis; and symptomatic controls, which were collected from participants with pain or other symptoms (excluding infertility) with a clinical suspicion of endometriosis, but no endometriosis lesions visualized by laparoscopy by experienced gynecological surgeons.

The case group comprised specimens for which the presence of endometriosis was diagnosed during laparoscopy and classified by the operating surgeon using the revised American Society of Reproductive Medicine (rASRM) stages (I: minimal; II: mild; III: moderate; IV: severe disease) [32]. Specimens were also grouped by disease subtype: peritoneal, ovarian, and deep infiltrating (DI) endometriosis.

\section{Disease specificity: participants \& sample collection}

Endometriosis cases and controls from the diagnostic accuracy assessment were utilized for the assessment of disease specificity and compared with residual plasma samples obtained from OBIO (CA, USA) and Ontario Tumour Bank (ON, Canada).

\section{Sample handling, processing \& mtDNA amplification Blood collection \& processing}

Whole blood was collected in 10-ml K2EDTA Vacutainers ${ }^{\circledR}$ (BD Medical [NJ, USA] p/n BD366643) and centrifuged within $1 \mathrm{~h}$ of collection at $2500 \times g$ for $10 \mathrm{~min}$ at $4^{\circ} \mathrm{C}$. The plasma layer was removed, aliquoted and stored at $-80^{\circ} \mathrm{C}$ until DNA extraction.

\section{DNA extraction}

We extracted total DNA from blood plasma $(200 \mu \mathrm{l})$ using the QIAamp 96 QIAcubeHT extraction kit (Qiagen, West Sussex, UK), automated on a QIAcube HT system (Qiagen) and eluted extracted DNA with buffer AE $(200 \mu \mathrm{l})$.

\section{$m t D N A$ deletion quantitative PCR \& quantitative PCR normalization with $18 s$ s $R$ A}

For both real-time PCR (quantitative PCR [qPCR]) procedures, we performed amplification in $20-\mu$ reactions using a 96-well microplate (Bio-Rad, Hertfordshire, UK), with each well containing un-normalized DNA template $(5 \mu \mathrm{l})$, SYBR ${ }^{\circledR}$ Green master mix and $250 \mathrm{nM}$ of each primer for the $8.7-\mathrm{kb}$ deletion and the $18 S \mathrm{rRNA}$. Primer sequences and master mix are trade secrets of MDNA Life Sciences Inc. We used a CFX96 Touch Real-time PCR Detection System (Bio-Rad) for qPCR with SYBR Green I fluorescence.

Cycling conditions for the $8.7-\mathrm{kb}$ deletion and $18 \mathrm{~S}$ rRNA were: 45 cycles of $30 \mathrm{~s}$ at $95^{\circ} \mathrm{C}, 30 \mathrm{~s}$ at $66^{\circ} \mathrm{C}$ and 30 $s$ at $72^{\circ} \mathrm{C}$. After amplification, we performed melting curve analysis from 70 to $90^{\circ} \mathrm{C}$, with a reading every $0.5^{\circ} \mathrm{C}$. Each plate of samples and controls was amplified in triplicate on three separate occasions.

\section{Standard PCR \& gel electrophoresis in DNA extracted from tissue}

DNA from $10 \mathrm{mg}$ of tissue was extracted following the QIAamp 96 QIAcubeHT extraction kit instructions on a QIAcube HT system and eluted with buffer AE $(200 \mu \mathrm{l})$. The eluate was diluted 1:100 with buffer AE. Standard qPCR was performed in 20- $\mu$ l reactions containing Accustart ${ }^{\text {TM }}$ II SuperMix (QuantaBio, MA, USA) and $250 \mathrm{nM}$ of each primer for the $8.7-\mathrm{kb}$ deletion and $5 \mu \mathrm{l}$ of DNA extract. Cycling conditions for the $8.7-\mathrm{kb}$ deletion and 
Table 1. Demographic and clinical characteristics.

\begin{tabular}{|c|c|c|c|}
\hline Characteristic & Total, n (\%) & Controls, n (\%) & Cases, n (\%) \\
\hline $\mathrm{N}$ & 182 & 32 & 150 \\
\hline Patient age ${ }^{\dagger}$ & $34.4( \pm 6.9)$ & $37.16( \pm 6.901)$ & $33.78( \pm 6.820)$ \\
\hline \multicolumn{4}{|l|}{ Hormone therapy status $\ddagger$} \\
\hline - Yes & $55(30.2)$ & $11(34.5)$ & $44(29.3)$ \\
\hline$-\mathrm{No}$ & $120(65.9)$ & $19(59.4)$ & $101(67.3)$ \\
\hline - Undetermined & $7(3.8)$ & $2(6.3)$ & $5(3.3)$ \\
\hline \multicolumn{4}{|l|}{ Menstrual phase ${ }^{\S}$} \\
\hline - No menstruation & $27(14.8)$ & $8(25.0)$ & $19(12.7)$ \\
\hline - Irregular menstruation & $15(8.2)$ & $2(6.3)$ & $13(8.7)$ \\
\hline - Menstruation & $30(16.5)$ & $6(18.8)$ & $24(16.0)$ \\
\hline - Follicular phase & $46(25.3)$ & $3(9.4)$ & $43(28.7)$ \\
\hline - Luteal phase and extended & $64(35.2)$ & $13(40.6)$ & $51(34.0)$ \\
\hline \multicolumn{4}{|c|}{ Non-endometriosis/endometriosis type } \\
\hline - Symptomatic & $18(9.9)$ & $18(56.3)$ & NA \\
\hline - Asymptomatic & $14(7.7)$ & $14(43.8)$ & NA \\
\hline - Peritoneal & $52(28.6)$ & NA & $52(34.7)$ \\
\hline - Ovarian & $48(26.4)$ & NA & $48(32.0)$ \\
\hline - Deep infiltrating & $50(27.5)$ & NA & $50(33.3)$ \\
\hline \multicolumn{4}{|l|}{ Endometriosis stage } \\
\hline - Stage I/II & NA & NA & $91(60.7)$ \\
\hline - Stage III/IV & NA & NA & $58(38.7)$ \\
\hline - Unknown & NA & NA & $1(0.7)$ \\
\hline \multicolumn{4}{|c|}{$\begin{array}{l}\text { †Mean (SD) is presented; mean and SD were calculated for patients that provided age at time of specimen collection. } \\
\text { ¥Patients' status within } 3 \text { months of specimen collection. } \\
\text { §Patients' menstrual status at time of specimen collection. } \\
\text { n: Number of patients/specimens; NA: Not applicable; SD: Standard deviation. }\end{array}$} \\
\hline
\end{tabular}

$18 \mathrm{r}$ rRNA were: 35 cycles of $30 \mathrm{~s}$ at $95^{\circ} \mathrm{C}, 30 \mathrm{~s}$ at $66^{\circ} \mathrm{C}$ and $30 \mathrm{~s}$ at $72^{\circ} \mathrm{C}$. Amplified DNA was ran on a $1.5 \%$ agarose gel. Electrophoresis was performed for $1 \mathrm{~h}$ at $80 \mathrm{~V}$.

\section{Quality control}

Quality control was performed as previously described [31].

\section{Rho 0 cell preparation}

Rho 0 cells were prepared as previously described $[31,33]$.

\section{Statistical analysis}

Statistical analysis was performed as previously described [31], except for this study GraphPad Prism 8.3 was used (GraphPad Software Inc., CA, USA).

\section{Results}

\section{Study population \& clinical specimens}

Demographics and clinical characteristics for participants who provided specimens are summarized in Table 1. Overall, the mean (standard deviation) ages of the control and case groups were statistically significantly different: 37.2 (6.9) and 33.8 (6.8) years; $\mathrm{p}=0.0124$. The majority of participants $(121 ; 66.5 \%)$ were not undergoing hormone therapy within the 3 months before specimen collection. Most participants who reported no menstruation were on hormones $(20 / 26 ; 76.9 \%)$.

Of the 182 specimens collected, 32 were from the control group, with 18 (9.9\%) from symptomatic participants and $14(7.7 \%)$ from asymptomatic participants. The remaining 150 specimens were from the case group in which $52(28.6 \%)$ participants had peritoneal, $48(26.4 \%)$ had ovarian and $50(27.5 \%)$ had DI endometriosis and were classified as $91(60.7 \%)$ with rASRM stage I/II disease and 58 (38.7\%) with stage III/IV. Of the 182 specimens 


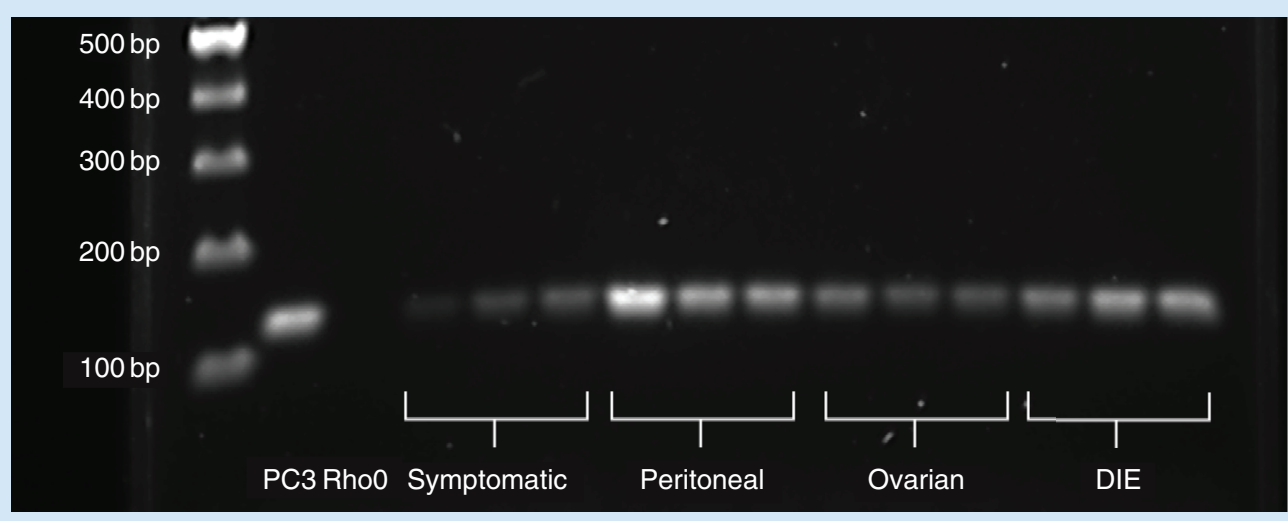

Figure 1. Polymerase chain reaction amplification of the $8.7-\mathrm{kb}$ deletion in normal endometrium and endometriosis lesions.

$178(97.8 \%)$ produced valid assay results, with two peritoneal and one ovarian endometriosis samples and one symptomatic control sample invalid for statistical analysis due to out of range $18 S \mathrm{rRNA}$ Cq.

\section{7-kb mtDNA deletion preliminary evaluation}

The 8.7-kb deletion was previously identified by these authors using a combination of next-generation sequencing (NGS) and proprietary data mining software in a set of ten cases and ten controls obtained from Fidelis Research (Sofia, Bulgaria). This deletion removes all or part of the genes between MT-ND2-5. We selected the 8.7-kb deletion for evaluation based upon sequence composition, the presence of a flanking repeat location within the major arc of the mitochondrial genome where proportionally more deletions are reported [34] and previous observation in endometriosis tissue lesions (Figure 1) using standard PCR. The marker has also been detected in these lesions using both qPCR and NGS sequencing (data not shown).

An initial round of standard (qualitative) PCR and visualization after gel electrophoresis was used to prequalify the deletion target and determine if the deletion: was detectable, had sufficient copy number for reliable detection, had the predicted amplicon size, and was specific and did not coamplify nuclear pseudogenes or generate nonspecific amplification products.

We successfully detected the deletion in circulating plasma and performed further evaluation by qPCR to determine whether the target was detectable in $r h o 0$ cells using more sensitive qPCR. We also evaluated if the assay had sufficient diagnostic accuracy and acceptable precision (defined as a maximum deviation of $1.5 \mathrm{Ct}$ between at least two of three replicates).

\section{Diagnostic accuracy of the 8.7-kb deletion}

Having successfully detected the 8.7-kb deletion in circulating plasma and endometriosis lesions, we investigated whether the $8.7-\mathrm{kb}$ deletion could discriminate between symptomatic controls versus all endometriosis; between the three subtypes; and between the revised American Society for Reproductive Medicine (r-ASRM) classification stages, in plasma from a larger set of clinical specimens. We performed the analyses using primarily the symptomatic controls and specimens from participants with confirmed disease to more accurately reflect the clinically relevant patient populations, that is, all presenting with symptoms of endometriosis. We also measured the frequency of the deletion in the asymptomatic control samples and did not detect a difference in the $8.7-\mathrm{kb}$ deletion between symptomatic and asymptomatic $(\mathrm{p}=0.681)$ control specimens.

\section{Symptomatic controls versus all disease}

We were able to discriminate well between symptomatic control and all endometriosis specimens using the 8.7$\mathrm{kb}$ assay. The area under the curve (AUC) $(95 \% \mathrm{CI})$ of $0.8007(0.7035-0.8979)$ was statistically significant $(\mathrm{p}<0.0001$; Figure 2). We examined receiver-operating curve coordinates and chose a threshold to optimize sensitivity, with a threshold of 6.65 discriminating between symptomatic controls and all subtypes/stages of endometriosis and acceptable sensitivity and specificity values (Table 2). 
Figure 2. Diagnostic accuracy of the 8.7-kb deletion: symptomatic versus all disease.

ROC: Receiver operating characteristic.

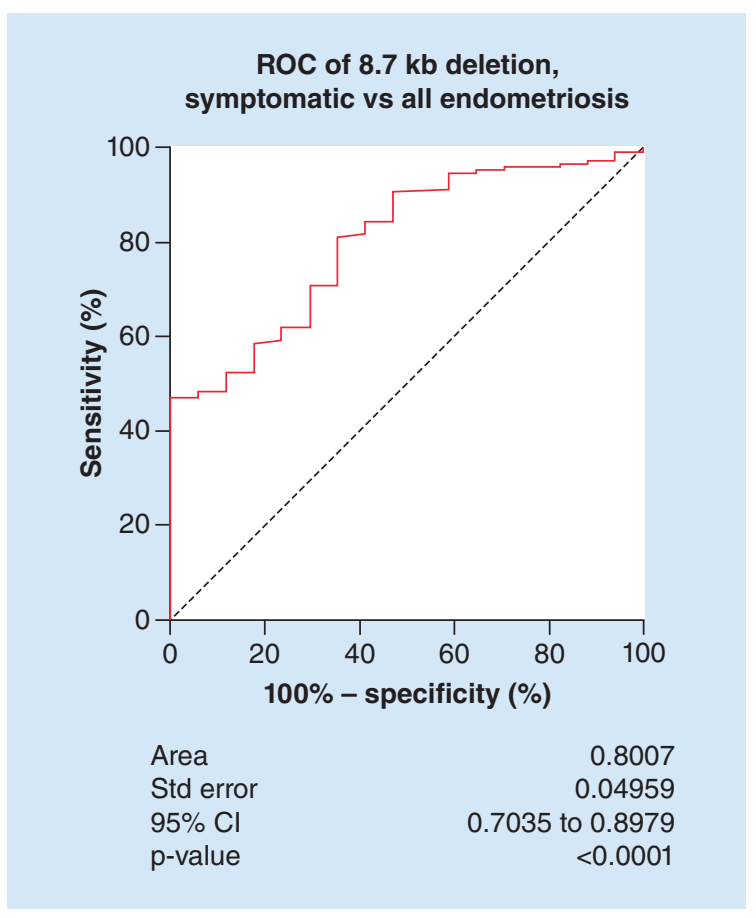

\begin{tabular}{|ll|}
\hline Table 2. Sensitivity of the 8.7-kb deletion at $65 \%$ specificity, cutoff 6.65. \\
\hline All disease & Sensitivity $(\%)[95 \% \mathrm{Cl}]$ \\
\hline Peritoneal & $80.95[73.85-86.48]$ \\
\hline Ovarian & $94.00[83.78-98.36]$ \\
\hline Deep infiltrating & $76.60[62.78-86.40]$ \\
\hline Stage I/II & $72.00[58.33-82.53]$ \\
\hline Stage III/IV & $86.52[77.00-92.12]$ \\
\hline
\end{tabular}

\section{Detection of disease by subtype}

It is important that we can accurately detect all disease subtypes of endometriosis. In our study, the 8.7-kb deletion assay differentiated between specimens from symptomatic controls and those from patients with peritoneal, ovarian, and DI endometriosis (Figure 3), with mean (SD) $\Delta$ Ct values of 6.724 (1.192) for asymptomatic controls, 6.908 (1.26) for symptomatic controls, 4.086 (2.134) for peritoneal disease, 5.283 (1.801) for ovarian disease and 5.617 (1.767) for DI endometriosis. Furthermore, the difference in normalized 8.7-kb deletion quantity between symptomatic controls was statistically significant for peritoneal $(p<0.0001)$, ovarian $(p=0.0002)$ and DI endometriosis $(\mathrm{p}=0.0023)$.

Diagnostic accuracy of the 8.7-kb deletion assay was also evaluated for each subtype (Figure 3). We accurately distinguished specimens from symptomatic controls and disease subtypes: AUC (95\% CI) was 0.8882 (0.80430.9722; $\mathrm{p}<0.0001)$ for detection of peritoneal disease, $0.7766(0.6572-0.8960 ; \mathrm{p}=0.0008)$ for ovarian and $0.7359(0.6057-0.8661 ; p=0.0039)$ for DI endometriosis. In addition, the threshold value of 6.65 gave acceptable sensitivity and specificity values for distinguishing between symptomatic controls versus peritoneal endometriosis, ovarian and DI disease (Table 2).

\section{Detection of disease by $r$-ASRM stage}

Endometriosis cases were classified into two stage groups: r-ASRM Stage I/II and Stage III/IV to determine if both low- and high-stage disease would be accurately identified using the 8.7-kb deletion. The 8.7-kb deletion assay differentiated between specimens from symptomatic controls and those from patients with low stage (Stage I/II) and high stage (Stage III/IV; Figure 4), with mean (SD) $\Delta \mathrm{Ct}$ values of 6.908 (1.26) for symptomatic controls, 


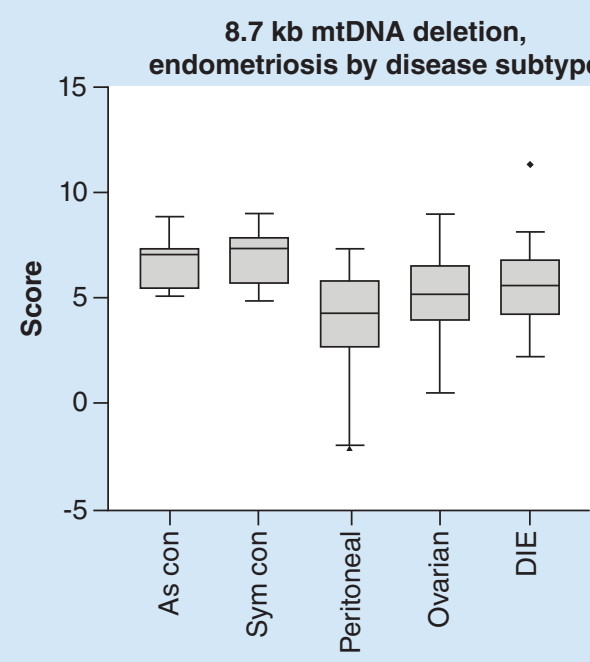

ROC of $8.7 \mathrm{~kb}$ deletion, symptomatic vs peritoneal

$\begin{array}{llllll} & \text { As. con. } & \text { Sym. con. Peritoneal } & \text { Ovarian } & \text { DIE } \\ \mathrm{n} & 14 & 17 & 50 & 47 & 50 \\ \text { Mean } & 6.724 & 6.908 & 4.086 & 5.283 & 5.617 \\ \text { Std deviation } & 1.192 & 1.26 & 2.134 & 1.801 & 1.767 \\ \text { Std error of mean } & 0.3185 & 0.3056 & 0.3018 & 0.2627 & 0.2499\end{array}$

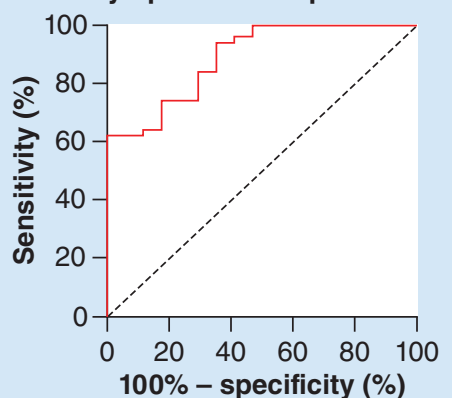

ROC of $8.7 \mathrm{~kb}$ deletion, symptomatic vs peritoneal

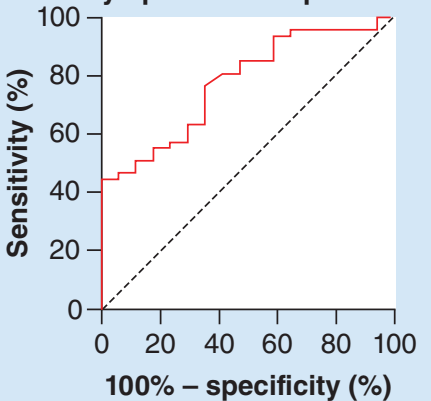

Area

Std error $\quad 0.0609$

$95 \% \mathrm{Cl} \quad 0.6572-0.8960$

$\mathrm{p}$-value

0.0008
ROC of $8.7 \mathrm{~kb}$ deletion,

symptomatic vs peritoneal

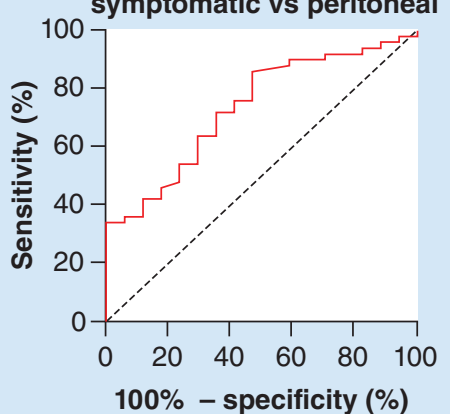

Area

Std error

95\% Cl

$\mathrm{p}$-value

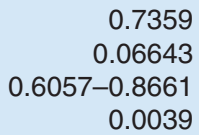

Figure 3. Distribution and diagnostic accuracy of the 8.7-kb deletion: detection of disease by subtype of endometriosis. As.: Asymptomatic; Con.: Control; ROC: Receiver operating characteristic; Sym.: Symptomatic.

4.614 (2.063) for low-stage and 5.565 (1.794) for high-stage disease.

Diagnostic accuracy assessed by receiver-operating curve was highest for Stage I/II: AUC $0.8361(0.7426-$ $0.9295 ; \mathrm{p}<0.0001)$ compared with Stage III/IV: AUC 0.7465 (0.6232-0.8697; p = 0.0021). At the threshold of 6.65 sensitivity and specificity for all stages was acceptable (Table 2 ).

Correlation with patient age, specimen age, hormonal therapy \& menstrual phase

For an ideal assay, diagnostic accuracy would not be affected by factors such as patient and specimen age, hormonal therapy and menstrual phase. We found no correlation between the $\Delta \mathrm{Ct}$ values and patient age $(p=0.749)$ nor specimen age by year of collection ( $\mathrm{p}=0.222$; Table 3 ). While there was a significant difference in the mean age of the case and control groups, a difference was not found when comparing the 8.7-kb deletion levels of those women greater than or equal to the mean age of the study population of 34 years and those who were less than 34 years: AUC $0.5213(0.4322-0.6103 ; \mathrm{p}=0.6404)$. Coupled with the lack of correlation between the deletion and patient age, it is unlikely to be a confounding variable in the assessment of diagnostic accuracy. No statistically significant differences in $\Delta \mathrm{Ct}$ values were seen when we stratified participants by hormonal status $(\mathrm{p}=0.838)$ or menstrual phase $(\mathrm{p}=0.233$; Table 3$)$. 


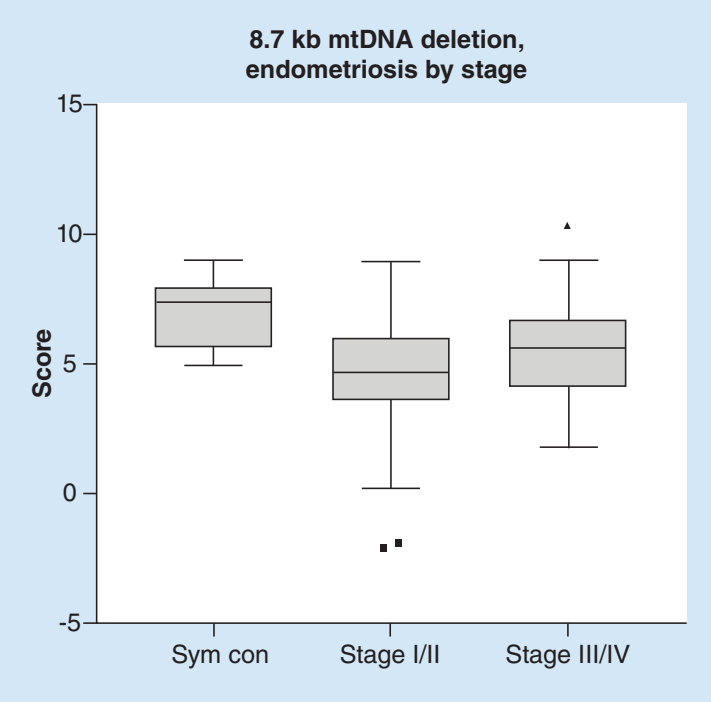

$\begin{array}{llll} & \text { Sym. con. } & \text { Stage I/II } & \text { Stage III/IV } \\ \mathrm{n} & 17 & 89 & 58 \\ \text { Mean } & 6.908 & 4.614 & 5.565 \\ \text { Std deviation } & 1.26 & 2.063 & 1.794 \\ \text { Std error of mean } & 0.3056 & 0.2187 & 0.2356\end{array}$
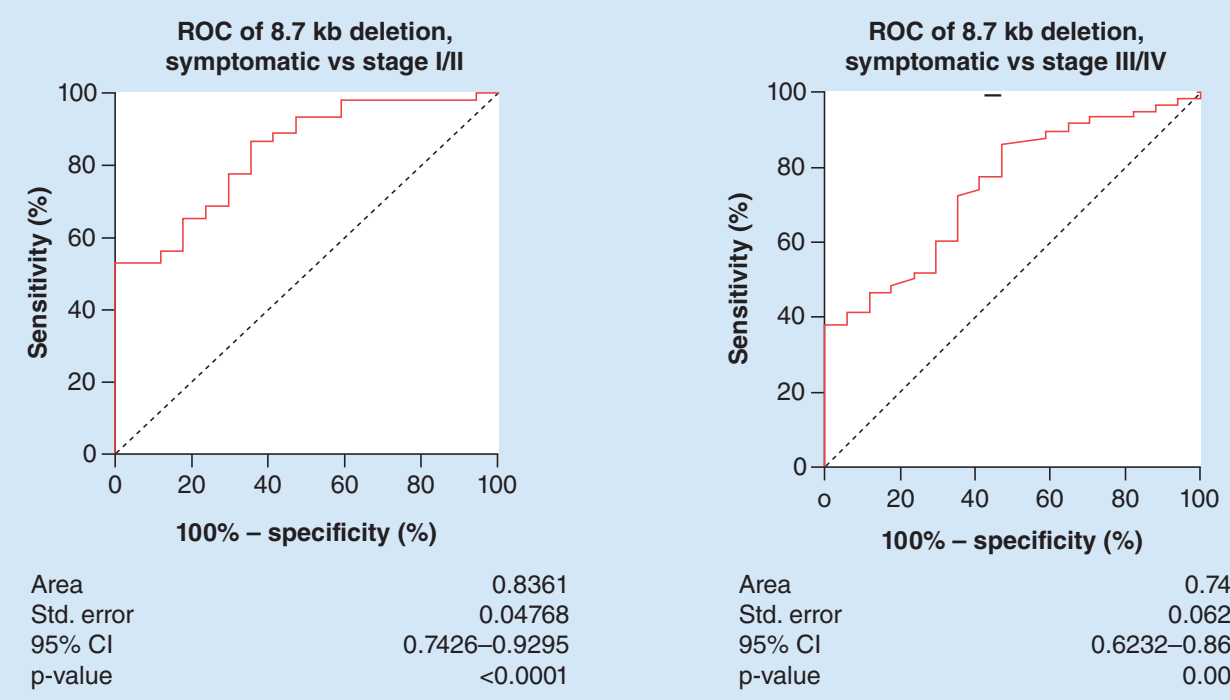

Area

Std. error

$95 \% \mathrm{Cl}$

p-value

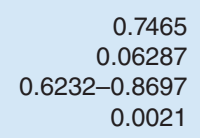

Figure 4. Distribution and diagnostic accuracy of the 8.7-kb deletion: detection of disease by stage of endometriosis. As.: Asymptomatic; Con.: Control; ROC: Receiver operating characteristic; Sym.: Symptomatic.

Table 3. Effect of participant and specimen age, hormonal therapy and menstrual cycle on the 8.7-kb assay performance.

\begin{tabular}{|c|c|c|c|c|c|c|}
\hline \multirow[t]{2}{*}{ Parameter } & \multicolumn{2}{|c|}{ Spearman correlation ( $r$ ) } & \multicolumn{2}{|c|}{ p-value } & \multicolumn{2}{|c|}{$\begin{array}{c}\text { Kruskal-Wallis test }{ }^{\mathbb{I}} \text { or Mann-Whitney } U \\
\text { test, }^{\S} \text { p-value }\end{array}$} \\
\hline & $\mathbf{D}^{\dagger}$ & $\mathrm{C}^{\ddagger}$ & D & C & D & C \\
\hline Patient age & -0.017 & -0.217 & 0.835 & .0242 & - & - \\
\hline Specimen age & -0.057 & 0.231 & 0.489 & 0.212 & - & - \\
\hline Hormonal status & - & - & - & - & 0.792 & 0.974 \\
\hline Menstrual cycle & - & - & - & - & 0.497 & 0.851 \\
\hline \multicolumn{7}{|c|}{$\begin{array}{l}\text { †Disease cases. } \\
\text { ¥Symptomatic controls. } \\
\S \text { The Mann-Whitney U test was used to determine effect of hormonal status on detection of endometriosis. } \\
\mathbb{I} \text { Kruskal-Wallis test was used to determine effect of menstrual cycle on detection of endometriosis. }\end{array}$} \\
\hline
\end{tabular}


Evaluating the disease specificity of the 8.7-kb deletion for endometriosis

To further assess whether other female diseases had elevated levels of the 8.7-kb deletion, we obtained plasma samples from women who were subsequently diagnosed with endometrial cancer $(\mathrm{n}=12)$, ovarian cancer $(\mathrm{n}=72)$ and breast cancer $(n=51)$ and compared the marker frequency to that of the three endometriosis subtypes (peritoneal, ovarian and DI endometriosis) as well as the symptomatic control group (Figure 4). Significantly, less 8.7-kb deletion was detected in all three cancers with endometrial cancer estimated as having 64-fold less deletion than endometriosis, ovarian cancer 16-fold less and breast cancer eightfold less $(\mathrm{p}<0.0001)$.

\section{Discussion}

In the present study, we demonstrated the utility of measuring the levels of 8.7-kb deletion biomarker in plasma samples as a potential assay for detecting endometriosis. Our assay met robustness criteria for diagnostic tests, utilized a minimally-invasive specimen from blood and accurately detected all subtypes and stages of disease, with best performance seen in the peritoneal subtype and low stages of endometriosis, both encountered at high frequency in a primary care setting. If successfully translated into clinical use, this assay could potentially shorten the time to diagnosis and enable medical intervention before surgery. Specimens are easy and inexpensive to collect via venipuncture, with a low possibility of co-morbidities associated with this type of collection.

Our research group previously reported the diagnostic accuracy of two other mitochondrial genome deletions as putative biomarkers of endometriosis [31]. These deletions, $1.2 \mathrm{~kb}$ and $3.7 \mathrm{~kb}$ in size also had good diagnostic accuracy within a like sample set, however, as a single marker the $8.7-\mathrm{kb}$ deletion has superior performance in the expected intended use population. Combining the three biomarkers achieves only a marginal increase in performance with a sensitivity of 83.21 at $65 \%$ specificity. The added cost, complexity and potential increase in the failure rate driven by the use of multiple targets does not support the inclusion of the lower performance markers in a commercial assay.

With good diagnostic accuracy, especially for low stage and peritoneal disease, the 8.7-kb deletion assay has the potential to augment the current standard of care, particularly in the diagnosis of peritoneal disease, which, unlike ovarian and DI endometriosis, cannot be reliably detected by imaging modalities. The relative simplicity of the 8.7-kb deletion assay means it could be viable for use in both primary and secondary care settings. Blood samples are routinely collected in primary care without the need for dedicated surgical space or equipment. The high copy number of mtDNA means standard DNA extraction methods can be used without enrichment techniques. Furthermore, a high failure rate for the test is unlikely given the ample amount of DNA recovered from a standard blood specimen. Real-time PCR-based technology is widely used in clinical laboratories producing easily interpreted and quantitative results.

In our study we showed an absence of correlation between the deletion and patient age, specimen age, hormone status or phase of menstrual cycle. A lack of correlation with menstrual stage simplifies testing requirements, with no need to consider menstruation phase when scheduling sample collection.

The significantly lower frequency of the $8.7-\mathrm{kb}$ deletion in breast, ovarian and endometrial cancer ranging from eightfold lower than endometriosis cases to undetectable in plasma provides some indication that this particular mutation is driven by nononcogenic processes. Unfortunately, the endometriosis status of these cancer cases are unknown so subgroup analyses is not possible; however, even an eightfold difference is readily delineated using a defined diagnostic cutoff based on Cq as each cycle in an efficient PCR such as this corresponds to a doubling of target DNA.

Endometriosis epidemiology is not yet well-characterized and the impact of significant preanalytical variables such as collection tube type, centrifugation speed and storage conditions can negatively impact effective comparison and compilation of datasets across various studies $[1,2,4,6]$. Notably, the plasma and tissue samples used in our studies have been collected according to standardized and publicly available methods, which we anticipate will facilitate more direct comparison to results of similar studies using the same protocols [27-30,35].

Recently, we have detailed the potential applications of mitochondrial biomarkers of endometriosis in the clinic [36]. Of paramount importance to the greatest number of women is the need to identify and treat the disease earlier in its pathogenesis, typically within the primary care setting. A quantitative diagnostic aid at this critical intervention point designed to identify as many disease positive women as possible (high sensitivity) complements well the relatively low-risk next step in the clinical pathway of introduction of medical therapies such as oral contraceptives or imaging to evaluate extent of disease. In this setting, a lower specificity in favor of a higher sensitivity is appropriate. Indeed, the incidence of endometriosis among females presenting with pelvic pain is as 
high as $87 \%$ [37], meaning in practice proportionally fewer women will be impacted. Indeed, the performance of the $8.7-\mathrm{kb}$ deletion for all types of endometriosis means that for every 100 women tested an estimated five may have false-positive test results with potential exposure to low-risk medical therapy, noninvasive imaging or specialist referral as a follow-up. Conversely, 70 women may now appropriately enter the endometriosis treatment pathway much more rapidly, realizing the first opportunity for truly earlier intervention. Furthermore, as the frequency of low stage and peritoneal disease near the time of first presentation is significantly higher in the primary care setting than in this study, an even greater benefit may be achieved in practice than illustrated here as a result of the 8.7-kb deletion's superior accuracy in these subgroups.

Our study had some limitations. Hormone and menstrual status were patient reported. The symptomatic control group was small as patients with surgical disconfirmation for endometriosis are difficult to obtain. Though these data are promising, we need to replicate and validate our observations using a larger dataset. Additional studies are needed to improve our understanding of the relationship between mitochondrial genome disruption and the pathogenesis of endometriosis.

\section{Conclusion}

Our assay, using the mitochondrial-derived $8.7-\mathrm{kb}$ deletion biomarker, is a potential, minimally invasive, bloodbased method for detecting endometriosis that may be translated for use in both primary and secondary clinical settings. A relatively simple and more patient-friendly approach may shorten the time to diagnosis, thereby improving the management of this debilitating condition and patients' quality of life.

\section{Future perspective}

Endometriosis is a complex disease with variable phenotypes and symptoms. However, in recent years, researchers have made significant progress in understanding and characterizing the disease-specific molecular pathways, in mapping out the epigenetics, and in identifying the genetic variants associated with the development of endometriosis. Furthermore, the standardization of sample processing and data analysis, prompted by the publication of guidelines from the World Endometriosis Research Foundation in 2014, has helped to reduce variability in patient history characterization and sample collection, enabling those dedicated to endometriosis research to develop tools needed to identify noninvasive diagnostic markers. In the future, we envisage mitochondrial genome biomarkers being used in the clinical setting, which will aid diagnosis of endometriosis and improve accuracy during differential diagnosis, thereby reducing the severity of disease experienced by patients and improving their quality of life.

\section{Summary points}

- Currently, surgery and histopathological confirmation are the gold standard methods for diagnosing endometriosis.

- Variable clinical presentation and nonspecific symptoms often result in delayed diagnosis and therefore, delayed treatment administration.

- Thus, there is a clear unmet need for reliable, less invasive diagnostic tools that also shorten the time to patients receiving appropriate medication.

- In our study, blood samples were prospectively collected from non-pregnant women, aged 18 years and over, scheduled to undergo laparoscopy for pelvic pain (symptomatic) or tubal ligation (asymptomatic).

- We applied our 8.7-kb deletion assay to accurately detect endometriosis in these samples, including all disease subtypes and stages.

- We found no correlation between disease detection and patient or specimen age, hormone therapy or menstrual phase.

- Mitochondrial genome-derived biomarkers, such as, the 8.7-kb deletion, offer the potential for minimally invasive, more patient-friendly diagnostic assays for diagnosing endometriosis that are conducive to a clinic setting.

- These biomarker assays may reduce the delay in diagnosis and improve endometriosis management. 


\section{Acknowledgments}

The authors are grateful to all women who participated in the ENDOX study at the Oxford Endometriosis CaRe Centre, and thank the research nurses C Hubbard, K Barrett and E Shepherd for their dedication and hard work in collecting biological samples and clinical data as part of the studies. The authors are grateful to $K$ Zondervan and $C$ Becker for providing access to the invaluable ENDOX study collections

\section{Financial \& competing interests disclosure}

This study is funded by MDNA Life Sciences Inc. A Harbottle, A Maggrah, R Usher, E Desa and J Creed are employed by MDNA Life Sciences Inc. and/or are inventors of intellectual property including patent applications relating to the information disclosed herein. The authors have no other relevant affiliations or financial involvement with any organization or entity with a financial interest in or financial conflict with the subject matter or materials discussed in the manuscript apart from those disclosed.

The authors acknowledge D Jordan of ICON, plc., who provided medical writing services in preparation of this manuscript. Funding for medical writing services was provided by MDNA Life Sciences, Inc

\section{Ethical conduct of research}

The authors state that they have obtained appropriate institutional review board approval or have followed the principles outlined in the Declaration of Helsinki for all human or animal experimental investigations. Written informed consent has been obtained from the participants involved.

\section{Open access}

This work is licensed under the Attribution-NonCommercial-NoDerivatives 4.0 Unported License. To view a copy of this license, visit http://creativecommons.org/licenses/by-nc-nd/4.0/

\section{References}

Papers of special note have been highlighted as: $\bullet$ of interest; $\bullet \bullet$ of considerable interest

1. Ozkan S, Murk W, Arici A. Endometriosis and infertility: epidemiology and evidence-based treatments. Ann. NY Acad. Sci. 1127, 92-100 (2008).

2. Rogers PA, D’Hooghe TM, Fazleabas A, et al. Defining future directions for endometriosis research: workshop report from the 2011 World Congress of Endometriosis in Montpellier, France. Reprod. Sci. 20(5), $483-499$ (2013).

3. Shafrir AL, Farland LV, Shah DK, et al. Risk for and consequences of endometriosis: a critical epidemiologic review. Best Pract. Res. Clin. Obstet. Gynaecol. 51, 1-15 (2018).

4. Endometriosis: an overview of Cochrane reviews. Cochrane Database Syst. Rev. 3, 1465-1858 (2014).

5. Giudice LC, Kao LC. Endometriosis. Lancet 364(9447), 1789-1799 (2004).

6. Vessey MP, Villard-Mackintosh L, Painter R. Epidemiology of endometriosis in women attending family planning clinics. BMJ 306(6871), 182-184 (1993).

7. Zondervan KT, Becker CM, Koga K, Missmer SA, Taylor RN, Viganò P. Endometriosis. Nat. Rev. Dis. Primer. 4(1), 9 (2018).

8. The Practice Committee of the American Society for Reproductive Medicine. Endometriosis and infertility. Fertil. Steril. 81, 1441-1446 (2004).

9. Vercellini P, Viganò P, Somigliana E, Fedele L. Endometriosis: pathogenesis and treatment. Nat. Rev. Endocrinol. 10, 261-275 (2014).

10. Nnoaham KE, Hummelshoj L, Webster P et al. Impact of endometriosis on quality of life and work productivity: a multicenter study across ten countries. Fertil. Steril. 96(2), 366.e8-373.e8 (2011).

11. Simoens S, Dunselman G, Dirksen C et al. The burden of endometriosis: costs and quality of life of women with endometriosis and treated in referral centres. Hum. Reprod. 27(5), 1292-1299 (2012)

12. Dunselman G a J, Vermeulen N, Becker C et al. ESHRE guideline: management of women with endometriosis. Hum. Reprod. 29(3), 400-412 (2014).

13. Hadfield R, Mardon H, Barlow D, Kennedy S. Delay in the diagnosis of endometriosis: a survey of women from the USA and the UK. Hum. Reprod. 11(4), 878-880 (1996).

14. Taylor HS. Diagnosing endometriosis: is laparoscopy the gold standard? Supplement to OBG Management. (2019).www.mdedge.com/obgyn/article/197704/endometriosis/diagnosing-endometriosis-laparoscopy-gold-standard

15. Ballard $\mathrm{K}$, Lowton $\mathrm{K}$, Wright J. What's the delay? A qualitative study of women's experiences of reaching a diagnosis of endometriosis. Fertil. Steril 86(5), 1296-1301 (2006).

16. Rifai N, Gillette MA, Carr SA. Protein biomarker discovery and validation: the long and uncertain path to clinical utility. Nat. Biotechnol. 24(8), 971-983 (2006). 
17. Hu C, van der Heijden R, Wang M, van der Greef J, Hankemeier T, Xu G. Analytical strategies in lipidomics and applications in disease biomarker discovery. J. Chromatogr. B Analyt. Technol. Biomed. Life Sci. 877(26), 2836-2846 (2009).

18. Liotta LA, Petricoin EF. Serum peptidome for cancer detection: spinning biologic trash into diagnostic gold. J. Clin. Invest. 116(1), 26-30 (2006).

19. Petricoin EF, Ardekani AM, Hitt BA et al. Use of proteomic patterns in serum to identify ovarian cancer. Lancet 359(9306), 572-577 (2002).

20. Petricoin EF, Belluco C, Araujo RP, Liotta LA. The blood peptidome: a higher dimension of information content for cancer biomarker discovery. Nat. Rev. Cancer 6(12), 961-967 (2006).

21. Zhang Z, Bast RC, Yu Y et al. Three biomarkers identified from serum proteomic analysis for the detection of early stage ovarian cancer. Cancer Res. 64(16), 5882-5890 (2004).

22. Anderson NL, Anderson NG. The human plasma proteome: history, character, and diagnostic prospects. Mol. Cell Proteomics 1(11), 845-867 (2002).

23. May KE, Conduit-Hulbert SA, Villar J, Kirtley S, Kennedy SH, Becker CM. Peripheral biomarkers of endometriosis: a systematic review. Hum. Reprod. Update 16(6), 651-674 (2010).

24. Fassbender A, Vodolazkaia A, Saunders P et al. Biomarkers of endometriosis. Fertil. Steril. 99(4), 1135-1145 (2013).

-. Review on recent work in biomarkers of endometriosis.

25. Ahn SH, Singh V, Tayade C. Biomarkers in endometriosis: challenges and opportunities. Fertil. Steril. 107(3), 523-532 (2017).

•• Review on recent work in biomarkers of endometriosis.

26. Rahmioglu N, Fassbender A, Vitonis AF et al. Harmonization of clinical and laboratory data to improve biomarker discovery in endometriosis: WERF EPHect. In: Biomarkers for Endometriosis. Springer, Cham, Switzerland, 199-226 (2017).

-• Summary of standardized methods in endometriosis research.

27. Rahmioglu N, Fassbender A, Vitonis AF et al. World Endometriosis Research Foundation Endometriosis Phenome and Biobanking Harmonization Project: III. Fluid biospecimen collection, processing, and storage in endometriosis research. Fertil. Steril. 102(5), 1233-1243 (2014).

- Harmonized methods for endometriosis research.

28. Fassbender A, Rahmioglu N, Vitonis AF et al. World Endometriosis Research Foundation Endometriosis Phenome and Biobanking Harmonisation Project: IV. Tissue collection, processing, and storage in endometriosis research. Fertil. Steril. 102(5), 1244-1253 (2014).

- Harmonized methods for endometriosis research.

29. Becker CM, Laufer MR, Stratton P et al. World Endometriosis Research Foundation Endometriosis Phenome and Biobanking Harmonisation Project: I. Surgical phenotype data collection in endometriosis research. Fertil. Steril. 102(5), 1213-1222 (2014).

- Harmonized methods for endometriosis research.

30. Vitonis AF, Vincent K, Rahmioglu N et al. World Endometriosis Research Foundation Endometriosis Phenome and Biobanking Harmonization Project: II. Clinical and covariate phenotype data collection in endometriosis research. Fertil. Steril. 102(5), 1223-1232 (2014).

- Harmonized methods for endometriosis research.

31. Creed J, Maggrah A, Reguly B, Harbottle A. Mitochondrial DNA deletions accurately detect endometriosis in symptomatic females of child-bearing age. Biomark. Med. 13(4), 291-306 (2019).

32. American Society for Reproductive Medicine. Revised American Society for Reproductive Medicine classification of endometriosis: 1996. Fertil. Steril. 67(5), 817-821 (1997).

33. King MP, Attardi G. Human cells lacking mtDNA: repopulation with exogenous mitochondria by complementation. Science 246(4929), 500-503 (1989).

34. Brandon MC, Lott MT, Nguyen KC et al. MITOMAP: a human mitochondrial genome database-2004 update. Nucleic Acids Res. 33 , D611-D613 (2005).

35. Casper RF. Introduction: new tools for enhancing collaborative endometriosis research. Fertil. Steril. 102(5), 1211-1212 (2014).

36. Creed JM, Maggrah A, Usher R, Desa E, Harbottle A. How can mitochondrial DNA deletions act as a biomarker for the detection of endometriosis within the clinic? Biomark. Med. (14(1), 5-8 2019).

-. Review of the utility of mitochondrial biomarkers in the clinic.

37. Davila GW, Kapoor D, Alderman E, Hiraoka M, Ghoniem GM, Peskin

BD. Endometriosis. Medscape (2018). https://emedicine.medscape.com/article/271899-overview 
